\title{
SPECTRAL PROPERTIES OF SOME POSITIVE OPERATORS IN A BANACH SPACE WITH THE DECOMPOSITION PROPERTY ${ }^{1}$
}

\author{
NAZAR H. ABDELAZIZ
}

\begin{abstract}
This paper presents further generalizations of some results due to Perron and Frobenius concerning the peripheral spectrum of finite matrices with nonnegative entries.
\end{abstract}

1. Introduction. Certain aspects in the Perron-Frobenius theory on matrices with nonnegative entries were generalized in [5] and [10] to the infinite dimensional situation. In [10] the investigation was carried out for operators defined on a Banach lattice, while in [5, 6.3], the cone of the underlying space was assumed to have interior elements. The purpose of this note is to indicate further extensions of these results to a larger class of spaces and operators.

In what follows, $(E,\|\cdot\|)$ is an ordered Banach space, i.e., a Banach space over $\mathbf{R}$ with a closed, normal and generating cone $K$. We shall assume, furthermore, that $E$ has the decomposition property. That is, whenever $x, y, z \in K$ and $z \leq x+y$, there exist $b_{1}, b_{2} \in K$ such that $z=b_{1}+b_{2}$, $b_{1} \leq x$ and $b_{2} \leq y$. We remark that the definition of an ordered Banach space given here differs from the one used in [13].

In $\$ 2$ we discuss the concept of an ideal in a space with the decomposition property. (This concept has also appeared in [3] in connection with simplex spaces.) It is shown that the closure of an ideal of the space $(E,\|\cdot\|)$ is again an ideal (Proposition 3). This will be particularly useful in $\$ 3$, where the class of irreducible operators is defined via the closed ideals of the space. The main result of [10], as well as Theorem 2 of [8], are extended to the present situation, while Theorem 6 , below, generalizes

Presented to the Society, June 19, 1972; received by the editors August 17, 1973. 46A40.

AMS (MOS) subject classifications (1970). Primary 47B55, 47A10; Secondary

Key words and phrases. The decomposition property, ordered space, positive cone, positive linear operator, peripheral spectrum.

1 This research is derived from the author's doctoral dissertion submitted to the University of Maryland. The author wishes to thank his advisor, Professor George J. Maltese, for the guidance provided during the preparation of that document. 
in part 6.3 of [5]. In the sequel, $E^{\prime}$ will denote the Banach dual space of $E$ and $K^{\prime}$ the dual cone of $K$. For further terminology and information see [11] and [13].

2. In [1] T. Ando gave a characterization of ordered Banach spaces which possess the decomposition property in terms of order properties of their duals. We state this result, which will be needed, in a slightly different form.

Theorem 1. Let $F$ be a Banach space with a closed cone $K$; then $F$ is an ordered Banach space with the decomposition property if and only if its Banach dual space is a locally convex lattice.

Proof. It need only be mentioned that if $F$ is an ordered Banach space with the decomposition property, then the lattice operations in $F^{\prime}$ are norm continuous. For if $B$ is the unit ball of $F$ there are positive scalars $\lambda, a$ such that $B \subseteq(\lambda \cdot B \cap K)-(\lambda \cdot B \cap K)$ and such that $0 \leq x \leq y, y \in B$, implies $x \in \alpha \cdot B$. This is because $K$ is both generating and normal. It follows directly from this and the definition of $|g|$ (for any $g \in F^{\prime}$ ) that there is a positive scalar $c$, such that whenever $|f| \leq|g|$ and $g \in B^{\circ}$, the polar of $B$ in $F^{\prime}$, then $f \in C \cdot B^{\circ}$.

By an ideal $I$ we shall mean a subspace with the following properties: (1) $-a \leq x \leq a, a \in I$, implies $x \in I$, (2) each $x \in I$ admits a decomposition $x=x_{1}-x_{2}$ with $x_{1}, x_{2} \in I \cap K$.

In the rest of this paper, $(E,\|\cdot\|)$ will denote an ordered Banach space with the decomposition property.

Lemma 2. There is a scalar $y>0$ such that for every ideal $J$ of $E$ and for each $x \in J, x$ admits a decomposition $x=x_{1}-x_{2}$, with $x_{i} \in J \cap K$ and $\left\|x_{i}\right\| \leq \gamma \cdot\|x\|(i=1,2)$.

Proof. Let $\lambda$ and $\alpha$ be as in the proof of Theorem 1 above, and suppose that $x$ lies in some ideal $J$. Then $x$ admits decompositions $x=x_{1}^{\prime}-x_{2}^{\prime}$, $x_{i}^{\prime} \in K,\left\|x_{i}^{\prime}\right\| \leq \lambda\|x\|(i=1,2)$ and $x=x_{1}^{\prime \prime}-x_{2}^{\prime \prime}, x_{i}^{\prime \prime} \in J \cap K(i=1,2)$, so we may write $0 \leq x_{1}^{\prime \prime}=x+x_{2}^{\prime \prime} \leq x_{1}^{\prime}+x_{2}^{\prime \prime}$. By the decomposition property there is $z_{1}, z_{2} \in K$ such that $z_{1} \leq x_{1}^{\prime}, z_{2} \leq x_{2}^{\prime \prime}$ and $x+x_{2}^{\prime \prime}=z_{1}+z_{2}$. From this it follows that $z_{1}=x+x_{2}^{\prime \prime}-z_{2} \in J \cap K$ and that $\left\|z_{1}\right\| \leq$ $\alpha \cdot\left\|x_{1}^{\prime}\right\| \leq \alpha \cdot \lambda \cdot\|x\|$. Put $x_{1}=z_{1}$ and $x_{2}=x_{1}-x$; then $x_{2}$ is also an element of $J \cap K$, and $\left\|x_{2}\right\| \leq(\alpha \lambda+1) \cdot\|x\|$.

Proposition 3. The closure of every ideal of $E$ is also an ideal. 
Proof. Let $\bar{I}$ denote the closure of an ideal $I$ of $E$, and suppose that $-x \leq y \leq x, x \in \bar{I}$. We want to show that $y \in \bar{I}$. Let $\left(x_{n}\right)$ be a sequence in $I$ converging to $x$, so that $x=x_{n}+u_{n}$, where $u_{n} \rightarrow 0$ as $n \rightarrow \infty$. Now there are sequences $\left(x_{n}^{\prime}\right) \subseteq I \cap K$ and $\left(u_{n}^{\prime}\right) \subseteq K$, such that $x_{n} \leq x_{n}^{\prime}, u_{n} \leq u_{n}^{\prime}$ and $\left\|u_{n}^{\prime}\right\| \leq \lambda \cdot\left\|u_{n}\right\|$ for all $n(\alpha, \lambda$ are as before $)$, so $0 \leq x+y \leq 2 x \leq$ $2 x_{n}^{\prime}+2 u_{n}^{\prime}$. The decomposition property now yields sequences $\left(z_{n}\right) \subseteq I$ and $\left(v_{n}\right), v_{n} \rightarrow 0$ as $n \rightarrow \infty$, such that $x+y=z_{n}+v_{n}$ for all $n$. Hence $y \in \bar{I}$. It remains to show that each $x \in \bar{I}$ has a decomposition $x=x_{1}-x_{2}$ with $x_{i} \in \bar{I} \cap K(i=1,2)$. Let $x \in \bar{I}$ and let $\left(x_{n}\right)$ be a sequence in $I$ such that $x=\Sigma x_{n}$. Applying Lemma $2, \Sigma x_{n}$ can be written as $\Sigma x_{n}^{\prime}-\Sigma x_{n}^{\prime \prime}$, where $\left(x_{n}^{\prime}\right)$, $\left(x_{n}^{\prime \prime}\right) \subseteq I \cap K$. This completes the proof.

3. In what follows, $T$ is a positive operator of $E$ (i.e., $T K \subseteq K$ ) whose spectral radius $r$ is a pole of the resolvent $R(\lambda, T)$ with residue $P$. Two classes of positive operators are considered here:

(1) An operator $T$ is irreducible if $T$ leaves invariant no closed ideals of $E$ except $\{0\}$ and $E$.

(2) An operator $T$ is strongly quasi-interior if for each $0 \neq x \in K$, there is a natural number $n_{0}\left(n_{0}=n(x)\right)$ such that $T^{n} x$ is a quasi-interior point for all $n \geq n_{0}$.

Using Proposition 3 and the fact that, for each $u \geq 0, \bigcup\{n[-u, u]$; $n=1,2, \cdots\}$ is an ideal, one can readily verify that the notion of irreducible operators defined above is equivalent to that of quasi-interior operators in [12]. In particular, every strongly quasi-interior operator is irreducible. It is also clear that when $K$ has a nonempty interior, then strongly quasi-interior and strongly positive (in [s]) are the same.

Let us recall that a subset $B$ of the complex domain is called cyclic if whenever $\alpha \in B$, with $\alpha=\lambda \gamma$ and $|\alpha|=\gamma$, then $\lambda^{n} \cdot \gamma \in B$ for all integers $n$. For any $x \in E,\langle x\rangle$ will denote the linear span of $x$. We now state the result concerning the peripheral spectrum of irreducible operators. This will extend the main theorem in [10].

Theorem 4. Let $E$ be an ordered Banach space with the decomposition property. Let $T$ be an irreducible operator of $E$ whose spectral radius $r$ is a pole of the resolvent of $T$. Then the peripheral spectrum of $T$ is of the form $r \cdot H$, where $H$ is the union of a finite number of cyclic subgroups of the unit circle, each of which is of finite order. Moreover, each element of $r \cdot H$ is a simple pole and a simple eigenvalue.

Proof. We note first that, because of the irreducibility of $T$, the spectral 
radius $r>0$ and $r$ is a simple pole of $T$. For simplicity we shall assume that $r=1$. Now $P$ is a positive projection of $E$ onto the fixed space of $T$. We observe that $\operatorname{dim} P E=1$. In fact, $P K$ is a nontrivial closed cone of the Banach space $P E$ such that every nonzero vector of $P K$ is quasi-interior in $P E$ [12, Theorem 2], [13, Lemma 1, $\$ 3$, Appendix]. Since every quasi-interior point is not a support point (see e.g. [11, p. 184]), the boundary of $P K$ in $P E$ contains no support points other than 0 . By applying Theorem 1 of [2], we have that the boundary of $P K$ in $P E$ consists only of $\{0\}$. Hence $\operatorname{dim} P E=1$ because $P K$ is a proper closed generating cone of $P E$.

Next, by Theorem 1 above (and [6, p. 20]), we may assume without loss of generality that $E^{\prime}$ is a Banach lattice, so $E^{\prime \prime}$ is also a Banach lattice. The second dual operator $T^{\prime \prime}$, is a positive operator of $E^{\prime \prime}$ whose spectral radius 1 is a pole of $R\left(\lambda, T^{\prime \prime}\right)$ with residue $P^{\prime \prime}$. Furthermore, $\operatorname{dim} P^{\prime \prime} E^{\prime \prime}=$ $\operatorname{dim} P^{\prime} E^{\prime}=\operatorname{dim} P E=1$. Therefore, it follows from [8, Theorem 2] and [12, Proposition 1] that the peripheral spectrum of $T^{\prime \prime}$ consists only of simple poles. Moreover, it is cyclic by $[7,4.10]$. Therefore the same is true for the peripheral spectrum of $T$.

It remains to show that every peripheral eigenvalue of $T$ is simple. We note first that, because $\operatorname{dim} P E=1$, there is an element $e$, quasi-interior to $K$, such that $P E=\langle e\rangle\left[12\right.$, Theorem 2]. Let $e^{\prime \prime}$ denote the image of $e$ in $E^{\prime \prime}$ and let $F$ be the closure of the lattice ideal generated by $e^{\prime \prime}$ in $E^{\prime \prime} . F$ is a Banach lattice which contains the canonical image of $E$ in $E^{\prime \prime}$. Furthermore, $F$ is $T^{\prime \prime}$-invariant since $T^{\prime \prime} e^{\prime \prime}=e^{\prime \prime}$. Let $S$ denote the restriction of $T^{\prime \prime}$ to $F$. The spectral radius of $S$ is 1 , and it is a simple pole of $R(\lambda, S)$ with residue $Q$ (where $Q$ is the restriction of $P^{\prime \prime}$ to $F$ ). Moreover, $Q F=\left\langle e^{\prime \prime}\right\rangle$.

It suffices to show that the peripheral eigenvalues of $S$ are simple. Consider the Banach lattice $\hat{F}=F / R$, where $R=\{x \in F: Q(|x|)=0\}$. Since $e^{\prime \prime}$ is a quasi-interior point in $F, \hat{e}$ (the canonical image of $e^{\prime \prime}$ in $\hat{F}$ ) is quasiinterior to the cone of $\hat{F}$. Now $R$ is an $S$-invariant, closed ideal of $F$. Hence $S$ (resp. $Q$ ) induces a positive operator $\hat{S}$ (resp. $\hat{Q}$ ) in the quotient space $\hat{F}$. Let us show that $\hat{S}$ is irreducible. Let $J \neq\{0\}$ be an $\hat{S}$-invariant, closed ideal of $\hat{F}$. Since 1 is a simple pole of $R(\lambda, \hat{S})$ with residue $\hat{Q}$, we have that $\hat{Q}=\lim _{\lambda \downarrow 1}(\lambda-1) R(\lambda, \hat{S})$. Hence $J$ is also $\hat{Q}$-invariant. Now $\hat{Q} \hat{F}=$ $\langle\hat{e}\rangle$; furthermore, it follows from the construction of $R$, that $0<\hat{Q} x$ whenever $0<x \in \hat{F}$. Hence $\hat{e} \in J$. Thus $J=\hat{F}$ since $\hat{e}$ is quasi-interior in $\hat{F}$.

Next, every eigenvector of $S$ that corresponds to a peripheral eigenvalue does not lie in $R$. In fact, if $x$ is such a vector then $S x=\alpha x ; \alpha \in \mathbf{C},|\alpha|=1$. Hence $|x|=|S x| \leq S(|x|)$. This implies that $|x| \leq Q(|x|)$. Thus $x \notin R$. 
Suppose now that $x, y$ are eigenvectors of $S$ associated to an $\alpha,|\alpha|=1$. The preceding argument shows that $\hat{x}, \hat{y}$ (the images of $x, y$ in $\hat{F}$ ) are eigenvectors of $\hat{S}$ associated to $\alpha$. Hence, by $[7,5.2] \hat{x}=c \cdot \hat{y}$ for some scalar $c$. However, this implies that $x=c \cdot y$; otherwise $x-c \cdot y$ would be an eigenvector of $S$ associated to $\alpha$ and which lies in $R$. This completes the proof.

The next proposition presents a relation between the irreducibility of the powers of an irreducible operator and the number of points in its peripheral spectrum. The example of an irreducible permutation matrix (in the finite dimensional case) shows that, in general, the powers need not be irreducible.

Proposition 5. Let $T$ be an irreducible operator of $E$ whose spectral radius $r$ is a pole of the resolvent of $T$. Then the following assertions are equivalent:

(i) $r$ is the only element in the peripheral spectrum of $T$;

(ii) $\lim \left(r^{-1} T^{n}\right)$ exists (with respect to the uniform topology of operators);

(iii) $T^{k}$ is irreducible for all $k \geq 1$.

Proof. We may take $r=1$. Let $P$ and $e$ be as in the proof of Theorem 4. (i) $\Rightarrow$ (ii) iș obvious because by Theorem $4, r$ is a simple pole.

(ii) $\Rightarrow$ (iii). We note first that $P x \neq 0$ whenever $0 \neq x \in K$, otherwise $\left(P^{-1}(0) \cap K\right)-\left(P^{-1}(0) \cap K\right)$ is a $T$-invariant closed ideal which is different from $\{0\}$ and, hence, would equal $E$. But then $P=0$, which is not true. Now by (ii), $\lim _{n \rightarrow \infty} T^{k n}=P$, and this shows that if $J \neq\{0\}$ is a $T^{k}$-invariant closed ideal, then it is also $P$-invariant. Therefore $e \in J$ so $J=E$.

(iii) $\Rightarrow$ (i). By Theorem 4 above and [4, Theorem 3] it is seen that $r\left(T^{k}\right)=1$ is a pole of $R\left(\lambda, T^{k}\right)$ for each $k \geq 1$. Suppose that $\alpha \neq 1$ is in the peripheral spectrum of $T$; then there is a positive integer $k$ such that $\alpha^{k}=1$ (Theorem 4 ) and so 1 is an eigenvalue of $T^{k}$ of multiplicity greater than 1 , contradicting Theorem 4 .

It is clear that if $T$ is strongly quasi-interior, then $T^{k}$ is irreducible for all $k \geq 1$; as a conséquence we obtain the following interesting result, which is a partial generalization of $[5,6.3]$.

Theorem 6. Let $T$ be a strongly quasi-interior operator of $E$, whose spectral radius $r$ is a pole of $R(\lambda, T)$. Then the peripheral spectrum of $T$ consists only of $r$.

The last proposition in this section extends [12, Theorem 2]. One could prove Proposition 7 by applying [12, Theorem 2] to the dual operator $T^{\prime}$. 
Proposition 7. Let $T$ be a positive operator on $E$ whose spectral radius is a pole of finite rank, then the peripheral spectrum of $T$ consists only of poles.

Remark. Clearly every Banach lattice is an ordered Banach space with the decomposition property. An example of I. Namioka [9] shows that the converse is not true. More generally, every simplex space is an ordered Banach space with the decomposition property, but not necessarily a vector lattice (see [3]).

Example. Consider the space $L^{p}(S, \Sigma, \mu)(1 \leq p<\infty) ; \mu$ is $\sigma$-finite, and let $L(s, t) \geq 0$ a.e. be a measurable function on $S \times S$ with

$$
\text { ess sup } \int_{S}|L(s, t)| d \mu(t) \leq M \quad \text { and } \quad \text { ess sup } \int_{S}|L(s, t)| d \mu(s) \leq M
$$

Then the operator $T f=g$, defined by the integral equation $g(s)=$ $\int_{S} L(s, t) f(t) d \mu(t)$, is a positive operator of $L^{p}$. If, moreover, there is an $n_{0}$ such that the $n$th iterate $L^{(n)}(s, t)>0$ a.e. for all $n \geq n_{0}$, then $T$ is strongly quasi-interior. A similar situation arises when dealing with infinite systems of linear equations (replacing $L^{p}$ by $l^{p}$ ).

\section{BIBLIOGRAPHY}

1. T. Andô, On fundamental properties of a Banach space with a cone, Pacific J. Math. 12 (1962) 1163-1169. MR 27 \#568.

2. E. Bishop and R. R. Phelps, The support functionals of a convex set, Proc. Sympos. Pure Math., vol. 7, Amer. Math. Soc., Providence, R. I., 1963, pp. 27-35. MR $27 \# 4051$.

3. E. G. Effros, Structure in simplexes, Acta Math. 117 (1967), 103-121. MR $34 \# 3287$.

4. M. A. Kaashoek and T. T. West, Locally compact monothetic semialgebras, Proc. London Math. Soc. (3) 18 (1968), 428-438. MR 37 \#3358.

5. M. G. Krein and M. Rutman, Linear operators leaving invariant a cone in a Banach space, Uspehi Mat. Nauk 3 (1948), no. 1 (23), 3-95; English transl., Amer. Math. Soc. Transl. (1) 10 (1962), 199-325. MR 10, 256.

6. H. J. Krieger, Beiträge zur Theorie positiver Operatoren, Akadamie-Verlag, Berlin, 1969.

7. H. P. Lotz, Über das Spektrum positiver Operatoren, Math. Z. 108 (1968), 15-32. MR $39 \# 1994$.

8. H. P. Lotz and H. H. Schaefer, Über einen Satz von F. Nirro und I.

Sawashima, Math. Z. 108 (1968), 33-36. MR 38 \#6382.

9. I. Namioka, Partially ordered linear topological spaces, Mem. Amer. Math. Soc. No. 24 (1957). MR 20 \#1193.

10. F. Niiro and I. Sawashima, On the spectral properties of positive irreducible operators in an arbitrary Banach lattice and problems of H. H. Schaefer, Sci. Papers College Gen. Ed. Univ. Tokyo 16 (1966), 145-183. MR 34 \#4919.

11. A. L. Peressini, Ordered topological vector spaces, Harper and Row, New York, 1967. MR $37 \# 3315$. 
12. H. H. Schaefer, Some spectral properties of positive linear operators, Pacific J. Math. 10 (1960), 1009-1019. MR 22 \#5893. \#1689.

13. - Topological vector spaces, Macmillan, New York, 1966. MR 33

DEPARTMENT OF MATHEMATICS, UNIVERSITY OF MARYLAND, BALTIMORE COUNTY, BALTIMORE, MARYLAND 21228

Current address: Department of Mathematics, Virginia Polytechnic Institute and State University, Blacksburg, Virginia 24061 